\title{
Spreading of Damage in the SK Spherical Spin Glass
}

\author{
Daniel A. Stariolo \\ Dipartimento di Fisica, Università di Roma I "La Sapienza" \\ and Istituto Nazionale di Fisica Nucleare, Sezione di Roma I \\ Piazzale Aldo Moro 2, 00185 Rome, Italy
}

January 17, 2014

\begin{abstract}
By considering the Langevin dynamics of the SK spin glass with a spherical constraint we calculate the asymptotic distance between two real replicas that evolve with the same thermal noise from different initial conditions. Despite the simplicity of the model its dynamics is known to be non trivial and presents aging phenomena. We found that the asympotic distance between two replicas is maximal for a whole family of initial conditions that are arbitrarily close at $t=0$. The asymptotic distance presents a dynamical transition at a temperature equal to the static phase transition one.
\end{abstract}




\section{Introduction}

The sensibility of the dynamics of a spins system with respect to the initial conditions has been extensively studied in the last years under the name of spreading of damage. This name was proposed in connection with biological systems where an important issue is the propagation of a little mutation or damage and its stability in an otherwise "normal" system (Kauffman 1969). In a spin system, information about the structure of phase space can be gained by observing the temporal evolution of the system from different initial conditions. Suppose we have two replicas of a system, $\sigma$ and $\tau$, and let them evolve in exactly the same external conditions (thermal noise, fields). The question then is: Are the long time dynamics of the two replicas sensible to small differences in initial conditions? This has been extensively studied in cellular automata (Martins, Verona de Resende, Tsallis and de Magalhães 1991, Hinrichsen, Weitz and Domany 1996), neural networks (Tamarit and Curado 1994), ferromagnetic Ising models (Stanley, Stauffer, Kertesz and Herrmann 1987, Derrida and Weisbuch 1987) and spin glasses (Derrida 1989). The quantities of interest are the Hamming distance at time $t$ between the two replicas:

$$
D(t)=\frac{1}{4 N} \sum_{i=1}^{N}\left(\sigma_{i}(t)-\tau_{i}(t)\right)^{2}
$$

and the probability $P(t)$ that the two replicas remain different after a time $t$. Different dynamical regimes or dynamical phase transitions can be seen by the change in the behaviour of $D(t)$ for $t \rightarrow \infty$. For example in the heat bath dynamics of a ferromagnet the distance goes to zero in the paramagnetic phase and it becomes different from zero below $T_{c}$ if the initial configurations of the replicas correspond to different magnetization states. A little damage always heals in a heat bath ferromagnet (Vojta 1996 a,b). This result has a trivial interpretation in terms of the structure of phase space.

In complex systems like spin glasses the phenomenology observed, mainly through computer simulations, is much more interesting (Derrida 1989). In general, three asymptotic regimes have been observed. A high temperature regime where the distance vanishes signalling a simple phase space with only one state down to a temperature $T_{1}$. This $T_{1}$ happens to coincide or be slightly smaller than the critical temperature $T_{c}$ of the pure ferromagnetic system. For $T_{2}<T<T_{1}$ the distance goes to a nonzero value that is independent of the initial conditions on the replicas. Finally for $T<T_{2}$ the distance goes to a nonzero value dependent on the initial conditions. This second temperature is near the spin glass transition temperature. Clearly this behaviour as $T$ is lowered reflects the increasing complexity of the phase space encountered by the replicas in their dynamics.

Extensive computer simulations done in spin glasses in dimensions 2, 3, 4 and 6 and mean field (Campbell and de Arcangelis 1991), and in spin glasses with asymmetric interactions (de Almeida, Bernardi and Campbell 1995), suggest that there is no dependence at all on the initial conditions, but this is probably because the simulations have not reached a sufficiently low temperature remaining at $T \geq T_{2}$.

Another important fact that may constrain the final output is the choice of the effective temperature of the initial conditions. Choosing random initial conditions that correspond to infinite temperature, the system will go through a rapid quench to the final temperature $T$. In this way it will select at random one of the accesible states at that temperature, 
probably remaining in those of higher energy for extremely long times. Other possibility is to initialize the replicas in equilibrium configurations at temperatures $T$ and $T^{\prime}$.

Despite the efforts done through extensive computer simulations, there are few analytic appoaches to the spreading of damage. Golinelly and Derrida (Golinelli and Derrida 1988) calculated the survival probability $P(t)$ for a mean field ferromagnet and Derrida (Derrida 1987) obtained the solutions for $D(t)$ in spin glasses with strongly diluted asymmetric interactions. Recently Vojta obtained interesting results for ferromagnets from a master equation approach in an effective field approximation (Vojta 1996 a,b). Trying to make contact with thermodynamic quantities, Coniglio, de Arcangelis, Herrmann and Jan (1989) have found relations between equilibrium spatial correlation functions and the (equilibrium) Hamming distance. Our aim in this work is to exploit the relation between the Hamming distance and dynamical, i.e. time dependent, correlation functions, as a way of extracting information of the precise dynamics of the spreading of damage process in complex systems. We have found analytic expressions for the distance $D(t)$ in a simple spin glass model, namely the SK model with a spherical constraint. This model is known to have a thermodynamic phase transition to a low temperature phase with replica symmetry (Kosterlitz, Thouless and Jones 1976). Thus it cannot be considered a "true" spin glass and its phase space structure can be analyzed with great detail, at least for $T \rightarrow 0$ (Kurchan and Laloux 1996). Despite its relative simplicity, a detailed study of the off equilibruim dynamics of the model (Cugliandolo and Dean 1995) revealed interesting properties like aging. We have studied the spreading of damage in this model finding some interesting peculiarities of its phase space as the exponential separation of some initially arbitrary near trajectories at zero temperature. On general grounds we have found a strong dependence of the final distance on the initial conditions and the absence of the intermediate regime that is consequence of the simple structure of phase space.

\section{The Model}

The spherical SK model is defined by the Hamiltonian:

$$
H=-\frac{1}{2} \sum_{i \neq j} J_{i j} \sigma_{i} \sigma_{j}
$$

where $\sigma_{i}, i=1 \ldots N$ are real valued spin variables that satisfy the constraint $\sum_{i=1}^{N} \sigma_{i}^{2}=N$. The couplings between the spins are given by the quenched random variables $J_{i j}$.

A possible dynamics for the model is defined by a Langevin equation which in the basis of the eigenvectors of the interaction matrix reads:

$$
\frac{\partial \sigma_{\mu}(t)}{\partial t}=(\mu-z(t)) \sigma_{\mu}(t)+\xi_{\mu}(t)
$$

where $\mu$ is the eigenvalue associated with the $\mu^{t h}$ eigenvector, $z(t)$ is a Lagrange multiplier enforcing the spherical constraint and $\xi_{\mu}(t)$ is a thermal white noise with zero mean and correlations:

$$
\left\langle\xi_{\mu}(t) \xi_{\nu}\left(t^{\prime}\right)\right\rangle=2 T \delta_{\mu \nu} \delta\left(t-t^{\prime}\right)
$$


$T$ is the temperature of the heat bath and $\langle\cdots\rangle$ means an average over the thermal noise.

The general solution of Eq.(3) is (Cugliandolo et al. 1995):

$$
\begin{aligned}
\sigma_{\mu}(t)= & \sigma_{\mu}\left(t_{0}\right) e^{\mu\left(t-t_{0}\right)} \exp \left\{-\int_{t_{0}}^{t} d \tau z(\tau)\right\}+ \\
& \int_{t_{0}}^{t} d t^{\prime \prime} e^{\mu\left(t-t^{\prime \prime}\right)} \exp \left\{-\int_{t^{\prime \prime}}^{t} d \tau^{\prime} z\left(\tau^{\prime}\right)\right\} \xi_{\mu}\left(t^{\prime \prime}\right)
\end{aligned}
$$

In this work we are interested in the time evolution of two real replicas of the system, $\sigma$ and $\tau$, that evolve with the same thermal noise. We then define the overlap between them at time $t$ :

$$
Q(t)=\frac{1}{N}\left[\sum_{i}\left\langle\sigma_{i}(t) \tau_{i}(t)\right\rangle\right]_{J}
$$

where $[\cdots]_{J}$ means average over the disorder variables $J_{i j}$. In the basis of the eigenvectors of $J$ it can be expressed as

$$
\begin{aligned}
Q(t) & \equiv\left[\left\langle\sigma_{\mu}(t) \tau_{\mu}(t)\right\rangle\right]_{J} \\
& =\int d \mu \rho(\mu)\left\langle\sigma_{\mu}(t) \tau_{\mu}(t)\right\rangle
\end{aligned}
$$

where $\rho(\mu)$ is the density of eigenvalues of the interaction matrix $J$. A common choice is an ensemble of matrices with independent gaussian elements with zero mean and variance proportional to $1 / N$. In the limit of large $N$ the eigenvalues of this ensemble are distributed according to the Wigner semicircle law:

$$
\rho(\mu)=\frac{1}{2 \pi} \sqrt{4-\mu^{2}} \quad \mu \in[-2,2] .
$$

Using the result for $\sigma_{\mu}(t)($ Eq.(6) $)$ (setting $\left.t_{0}=0\right)$ one obtains an expression for the overlap

$$
Q(t)=\frac{1}{\sqrt{\Gamma_{\sigma}(t) \Gamma_{\tau}(t)}}\left[\sigma_{\mu}(0) \tau_{\mu}(0) e^{2 \mu t}+2 T \int_{0}^{t} d t^{\prime} \sqrt{\Gamma_{\sigma}\left(t^{\prime}\right) \Gamma_{\tau}\left(t^{\prime}\right)} e^{2 \mu\left(t-t^{\prime}\right)}\right]_{J}
$$

where $\Gamma(t) \equiv \exp \left(2 \int_{0}^{t} d t^{\prime} z\left(t^{\prime}\right)\right)$. Using the spherical constraint $\Gamma(t)$ can be computed as the solution of a Volterra integral equation of the second kind (Cugliandolo et al. 1995):

$$
\Gamma(t)=\left[\left(\sigma_{\mu}(0)\right)^{2} e^{2 \mu t}+2 T \int_{0}^{t} d t^{\prime} \Gamma\left(t^{\prime}\right) e^{2 \mu\left(t-t^{\prime}\right)}\right]_{J}
$$

The spherical constraint implies $\Gamma(0)=1$.

By solving the closed equations (9) and (10) for different sets of initial conditions and using the spherical constraint we can finally calculate the distance between the two replicas at time t as:

$$
D(t)=\frac{1-Q(t)}{2}
$$

which is a direct generalization of the Hamming distance commonly used in binary systems. 


\section{Results}

We have studied the asymptotic behaviour of the distance $D(t)$ for three classes of initial conditions, namely:

1. Opposite initial conditions: $\sigma_{\mu}(0)=-\tau_{\mu}(0)$.

2. Independent initial conditions: $\left[\sigma_{\mu}(0) \tau_{\mu}(0)\right]_{J}=0$.

3. Arbitrarily correlated initial conditions. This class includes as particular case initial conditions that differ by an arbitrarily small fraction of spins as in the original defintion of damage.

In the first two classes we can even distinguish the initial conditions in two additional sub-classes:

- Uniform initial conditions: $\sigma_{\mu}(0)=1, \forall \mu$. This has the same projection onto each eigenvector of $J$ and corresponds to a random configuration in the original basis.

- Staggered initial conditions: $\sigma_{\mu}(0)=\sqrt{N} \delta_{a \mu}$. A condition of this type has a finite projection onto only one eigenvector of $J$.

We now proceed to present the results for each case.

\subsection{Opposite Initial Conditions}

First of all we can see immediatly that if $\sigma_{\mu}(0)=\tau_{\mu}(0)$ then $\Gamma_{\sigma}=\Gamma_{\tau}$ and the distance is zero for all $\mathrm{t}$, as it must. Now if $\sigma_{\mu}(0)=-\tau_{\mu}(0)$ we still have $\Gamma_{\sigma}(t)=\Gamma_{\tau}(t) \equiv \Gamma(t)$, but from Eqs.(9) and (10)

$$
Q(t)=1-\frac{2}{\Gamma(t)}\left[\left(\sigma_{\mu}(0)\right)^{2} e^{2 \mu t}\right]_{J}
$$

\subsubsection{Uniform initial condition}

In this case we have :

$$
\left[\left(\sigma_{\mu}(0)\right)^{2} e^{2 \mu t}\right]_{J}=\frac{I_{1}(4 t)}{2 t}
$$

and

$$
\Gamma(t)=\frac{1}{T} \sum_{k=0}^{\infty} k \frac{I_{k}(4 t)}{2 t} T^{k} \quad T<1 .
$$

where $I_{k}(x)$ is the modified Bessel function of order $k$. For large arguments $I_{k}(x) \approx$ $e^{x} / \sqrt{2 \pi x}$ and $Q(t)$ tends to the asymptotic value $\lim _{t \rightarrow \infty} Q(t) \equiv Q_{\infty}=1-2(1-T)^{2}$. Consequently the asymptotic distance between the two initially opposite replicas will be:

$$
D_{\infty}=(1-T)^{2}=q_{E A}^{2}
$$

where $q_{E A}$ is the Edwards-Anderson order parameter that equals $1-T$ in the spherical spin glass (Kosterlitz et al. 1976). At $T=0$ the distance is frozen and $D(t)=D(0)=1$ for all 
times. This can be interpreted within a geometric description of phase space (Kurchan et al. 1996): the energy has two absolute minima parallel and antiparallel in the direction of the maximum eigenvalue of $J_{i j}, \mu=2$. Phase space is divided in two equivalent halves that are geometrically identical and form the basins of attraction of the two minima. Consequently two opposite uniform initial conditions will evolve to the two distinct minima encoutering in the evolution the same landscape and so preserving the distance along the way towards the minima, which in turn dominate the asymptotic dynamics.

As $T$ grows the thermal fluctuations permit the replicas to come closer to each other up to a value equal to the square of the self-overlap and finally merge when $T$ goes to one signalling the transition to a high temperature phase characterized by $D=0$, that means the system has a unique pure state.

\subsubsection{Staggered initial condition}

In this case $\left[\left(\sigma_{\mu}(0)\right)^{2} \exp (2 \mu t)\right]_{J}=\exp (2 a t) . \quad \Gamma(t)$ can't be expressed in a simple closed form as in the previous case so we only quote its asymptotic form that depends on the value of $a$. If $a=2$ (the initial condition has a nonvanishing projection onto the maximum eigenvalue) then $\Gamma(t) \approx \exp (4 t) /(1-T)$ and

$$
D_{\infty}=1-T=q_{E A}
$$

The distance decreases linearly with $T$. Comparing with the previous case, even though in both cases the initial conditions are opposite, when the systems start from a random configuration the final distance is smaller than when they start aligned in the direction of the maximum eigenvalue. As was noted by Kurchan et al. (1995), at finite temperature the component in the direction of the maximum eigenvalue has a finite probability of changing sign at any time unless the initial condition is deep in a basin, this fact may be responsible for the different asymptotic distance between uniform and staggered $(a=2)$ initial conditions.

If $a<2, \Gamma(t) \approx \frac{T}{(1-T)^{2}} \frac{1}{2-a} \frac{1}{\sqrt{4 \pi}} \frac{\exp (4 t)}{(2 t)^{3 / 2}}$ and the overlap asymptotically behaves as

$$
Q(t) \approx 1-4 \sqrt{\pi} \frac{(1-T)^{2}}{T}(2-a)(2 t)^{3 / 2} e^{(2 a-4) t},
$$

so it relaxes exponentially to 1 . This means that in the infinite time limit the distance between the replicas goes to zero for all $T>0$. This again can be attributed to the fact that the initial conditions are not deep in the basins of the minima and in this case , although opposite, have in fact zero initial projection on them.

If $T=0$ we can see from (9), (10) and (11) that $D(t)=1$ at all times (the distance is frozen). Staggered initial conditions are fixed points of the dynamics at zero temperature and consequently the distance between them remains maximal.

\subsection{Independent initial conditions}

Independent initial conditions satisfy $\int d \mu \rho(\mu) \sigma_{\mu}(0) \tau_{\mu}(0)=0$. We have studied two cases, one with staggered initial conditions $(a<2)$ in both replicas and other with uniform projection on a subset of the eigenvectors of $J$ in such a way as to preserve independence between 
the initial configurations. In both cases $\left[\sigma_{\mu}(0) \tau_{\mu}(0) \exp (2 \mu t)\right]_{J}=0$ and the equation for the overlap reads:

$$
Q(t)=\frac{1}{\sqrt{\Gamma_{\sigma}(t) \Gamma_{\tau}(t)}} 2 T \int_{0}^{t} d t^{\prime} \sqrt{\Gamma_{\sigma}\left(t^{\prime}\right) \Gamma_{\tau}\left(t^{\prime}\right)}\left[e^{2 \mu\left(t-t^{\prime}\right)}\right]_{J}
$$

\subsubsection{Staggered initial conditions}

Let's consider staggered initial conditons in both replicas:

$$
\begin{aligned}
& \sigma_{\mu}(0)=\sqrt{N} \delta_{a \mu} \\
& \tau_{\mu}(0)=\sqrt{N} \delta_{b \mu} \quad a \neq b \neq 2 .
\end{aligned}
$$

In this case $\left[\left(\sigma_{\mu}(0)\right)^{2} \exp (2 \mu t)\right]_{J}=\exp (2 a t)$ and $\left[\left(\tau_{\mu}(0)\right)^{2} \exp (2 \mu t)\right]_{J}=\exp (2 b t)$. We have not been able to solve the integral for the overlap Eq.(18) analytically but we have done the integration numerically (Linz 1985). The result for the asymptotic distance as a funtion of temperature is shown in Fig.(1) for two arbitrary initial conditions in different basins.

Again at $T=0$ the dynamics is frozen and $D(t)=D(0)=0.5$. Note that in the case of opposite initial conditions the initial overlap was $Q(0)=-1$ and now it is $Q(0)=0$. At an arbitrarily small but finite temperature the final distance falls down to small values and already for $T \geq 0.05$ we have $D_{\infty} \leq 0.1$. This means that although the initial configurations are uncorrelated they finally become very near each other, although they do not completely merge as was the case with opposite initial conditions.

\subsubsection{Piecewise uniform initial conditions}

We choose here initial conditions of the form:

$$
\sigma_{\mu}(0)=\left\{\begin{array}{lll}
\kappa & \text { if } & \mu<c \\
0 & \text { if } & \mu \geq c
\end{array}\right.
$$

and

$$
\tau_{\mu}(0)=\left\{\begin{array}{lll}
0 & \text { if } & \mu<c \\
\lambda & \text { if } & \mu \geq c
\end{array}\right.
$$

The values of $\kappa$ and $\lambda$ depend on $c$ through the spherical constraint: $\int_{-2}^{c} d \mu \rho(\mu) \kappa^{2}=1$ and $\int_{c}^{2} d \mu \rho(\mu) \lambda^{2}=1$. For simplicity we have chosen $c=0$ so that $\kappa^{2}=\lambda^{2}=2$. For the Gamma functions we obtained the following equations:

$$
\begin{aligned}
& \Gamma_{\sigma}(t)=e^{-2 t} \frac{I_{1}(2 t)}{t}+2 T \int_{0}^{t} d t^{\prime} \Gamma_{\sigma}\left(t^{\prime}\right)\left[e^{2 \mu\left(t-t^{\prime}\right)}\right]_{J} \\
& \Gamma_{\tau}(t)=2\left(\frac{I_{1}(4 t)}{2 t}-\frac{e^{-2 t}}{2} \frac{I_{1}(2 t)}{t}\right)+2 T \int_{0}^{t} d t^{\prime} \Gamma_{\tau}\left(t^{\prime}\right)\left[e^{2 \mu\left(t-t^{\prime}\right)}\right]_{J}
\end{aligned}
$$

We have solved equations (21), (22) and (18) numerically and the result for the long time limit is shown in Fig.(2).

The final distance decays with temperature more like in the case of opposite initial conditions than in that of independent staggered ones, at each temperature the distance 
is greater than for staggered configurations. It may be noted that while in the case of staggered configurations both initial states would lead to non-equilibrium dynamics (zero projection on the maximum eigenvalue of $J$ ), in this case one replica has a component in the direction $a=2$ while the other has not.

\subsection{Arbitrarily correlated initial conditions}

As a final class of initial conditions we choose a pair that is arbitrarily close at $t=0$. We considered $\sigma_{\mu}(0)=1 \forall \mu$, i.e. a uniform initial condition in the firt replica and for the other we choose

$$
\tau_{\mu}(0)= \begin{cases}\kappa & \text { if } \quad-2 \leq \mu \leq 2-\epsilon \\ 0 & \text { if } \quad 2-\epsilon<\mu \leq 2\end{cases}
$$

In this form both initial configurations are equal if $\epsilon=0$ and have a decreasing correlation as $\epsilon$ grows $(0 \leq \epsilon<4)$. The value of $\kappa$ as a function of $\epsilon$ can be determined exactly from the spherical constraint. It turns to be $\kappa^{2}=2 \pi /[\pi+2 \arcsin (1-\epsilon / 2)+(1-\epsilon / 2) \sqrt{\epsilon(4-\epsilon)}$. Now we have to solve the full equations (9) and (10). We note that in this case the behaviour of $Q(t)$ at $T=0$ is different from the previous cases. For correlated initial conditions the distance is not frozen even at zero temperature. The expressions for the $\Gamma(t)^{\prime} s$ and $Q(t)$ simplify to:

$$
\begin{aligned}
\Gamma_{\sigma}(t) & =\left[e^{2 \mu t}\right]_{J} \\
\Gamma_{\tau}(t) & =\left[\left(\sigma_{\mu}(0)\right)^{2} e^{2 \mu t}\right]_{J} \\
Q(t) & =\frac{\left[\sigma_{\mu}(0) \tau_{\mu}(0) e^{2 \mu t}\right]_{J}}{\sqrt{\Gamma_{\sigma}(t) \Gamma_{\tau}(t)}}
\end{aligned}
$$

It is straightforward to see that

$$
\begin{aligned}
Q(t) & =Q(0) \sqrt{\frac{\Gamma_{\tau}(t)}{\Gamma_{\sigma}(t)}} \\
& =\frac{1}{\kappa} \sqrt{\frac{\Gamma_{\tau}(t)}{\Gamma_{\sigma}(t)}}
\end{aligned}
$$

Consequently if $\epsilon \neq 0, Q(t)$ evolves in time even at $T=0$. An explicit calculation shows that for any $\epsilon \neq 0, \Gamma_{\tau}(t)$ goes asymptotically to zero while $\Gamma_{\sigma}(t)$ grows exponentially for long times so that the overlap decays exponentially fast and the final distance is always maximal $D(t) \rightarrow 0.5$. This is somewhat surprising as it indicates that configurations with arbitrary initial correlation and deterministic dynamics decorrelate completely exponentially fast, something reminiscent of chaotic dynamics. In fact this behaviour can be understood looking at the geometry of phase space. At zero temperature the dynamics is deterministic and the asymptotic evolution dominated by the maximum eigenvalue. Consequently, a uniform initial condition will evolve towards a minimum. The initial condition on the other replica has zero projection onto the minima and its dynamics will evolve towards the direction of the maximum eigenvalue onto which it has an initial nonzero component. 
Asymptotically it will reach a fixed point that will correspond, in general, to a critical point of high order and orthogonal to the minima, then the distance will be maximal. Conversely, a similar calculation considering two initial conditions with finite projection on the maximum eigenvalue direction shows that the configurations asymptotically merge and the distance goes to zero.

For the case of finite temperature we have solved the full equations numerically and the result, for initial conditions of the form (23), is identical to that of Fig.(2) corresponding to independent uniform initial conditions. So, even at finite temperature, the distance grows and reaches a finite asymptotic value that depends on the temperature and is independent of $\epsilon$. It can be said that a little initial "damage" in a configuration propagates with time, the phase is "active".

$\epsilon$ introduces a time scale for the decay of the correlations. In general the overlap enters its asymptotic regime in a time that scales as $t \approx 1 / \epsilon$. As $\epsilon$ becomes smaller the initial conditions have a larger correlation and the times needed for reaching the asymptotic regime grow as $\epsilon^{-1}$.

When both configurations present an initial projection in the direction of the maximum eigenvalue the asymptotic distance vanishes also in the presence of thermal noise and a little damage finally dissapears.

\section{Conclusions}

We have studied analytically the spreading of damage in the spherical SK model with Langevin dynamics. With respect to the general spreading of damage phenomenology, we have found that in the low temperature phase of the model the long time distance between the states of two real replicas of the system depend on the initial conditions on each replica. In this model the intermediate phase as described in the introduction is absent and the dynamic transition temperature at which the asymptotic distance begins to be non zero coincides with the static one.

The results for zero temperature can be readily interpreted in terms of the geometry of phase space. The most interesting result is that two states that are initially arbitrarily close will flow to a maximal distance if at least one of them has vanishing initial projection onto the direction of the maximum eigenvalue. Conversely, if both have an initial component on the direction of $\mu=2$ the trajectories will finally merge independently of the initial correlation.

For the other classes of initial conditions considered we found that opposite or independent ones are fixed points for the dynamics of the distance and are in this sense less interesting.

At finite temperature the asymptotic distances are simple functions of $q_{E A}$ for those initial conditions that lead asymptotically to equilibrium dynamics, i.e. those which present an initial condensation in the direction of the maximum eigenvalue. In these cases, when the inital configurations are arbitrarily close to each other they finally merge giving an asymptotic zero distance.

A final comment regards the particular choice for the dynamics. It is known from the literature on spreading of damage that different dynamics can lead to different results 
and so the conclusions can be model dependent. The present calculations for Langevin dynamics could be extended to Monte Carlo dynamics following the lines of a recent work (Bonilla, Padilla, Parisi and Ritort 1996). In that work it is enphasized the importance of the acceptance rate in the off-equilibrium dynamics of the system. For asymptotically long times, those which we are interested in this approach, both dyamics are equivalent and one expects that they will give the same results. The scale of times in which both dynamics are comparable depends on the acceptance rate of the Metropolis algorithm.

The present analysis can be extended to more complicated systems which are expected to have a richer phenomenology. The natural extension is to consider the p-spin spherical model for $p>2$. The study of these models is in progress.

Acknowledgments I wish to thank D. Dean and J. Kurchan for discussions and comments. 


\section{Figure Captions}

Figure 1: Asymptotic distance vs. temperature for staggered independent initial conditions with $a=-0.2$ and $b=1.5$ (see text).

Figure 2: Asymptotic distance vs. temperature for piecewise uniform independent initial conditions. 


\section{References}

Bonilla L L, Padilla F G, Parisi G and Ritort F 1996, cond-mat 9602147.

Campbell I. A. and de Arcangelis L. 1991 Physica A 178, 29.

Coniglio A., de Arcangelis L., Herrmann H.J. and Jan N. 1989 Europh. Lett. 8, 315.

Cugliandolo L F and Dean D S 1995 J. Phys. A: Math.Gen. 28, 4213.

de Almeida R.M.C., Bernardi L. and Campbell I.A. 1995 J. Phys. I France 5, 355.

Derrida B. 1987 J. Phys. A: Math.Gen. 20, L721.

Derrida B. and Weisbuch G 1987 Europhys.Lett. 4, 657.

Derrida B. 1989 Phys. Rep. 184, 207.

Golinelli O. and Derrida B. 1988 J. Phys. France 49, 1633.

Hinrichsen H, Weitz J S and Domany E 1996 preprint cond-mat 9611085.

Kauffman S. A. 1969 J. Theor. Biol. 22, 437.

Kosterlitz J M, Thouless D J and Jones R C 1976 Phys. Rev. Lett. 36, 1217.

Kurchan J and Laloux L 1996 J. Phys. A: Math.Gen. 29,1929.

Linz P 1985 Analytical and Numerical Methods for Volterra Equations (SIAM Studies in Applied Mathematics, Philadelphia).

Martins M L, Verona de Resende H F, Tsallis C and de Magalhães A C N 1991 Phys.

Rev. Lett. 66, 2045.

Stanley H E, Stauffer D, Kertesz J and Herrmann H J 1987 Phys. Rev. Lett. 59, 2326.

Tamarit F A and Curado E M F 1994 J. Phys. A 27,671.

Vojta T. Damage spreading and dynamical stability of kinetic Ising models, cond-mat

9610084; Chaotic behaviour and damage spreading in the Glauber Ising model - a master equation approach, cond-mat 9611232. 
Figure 1

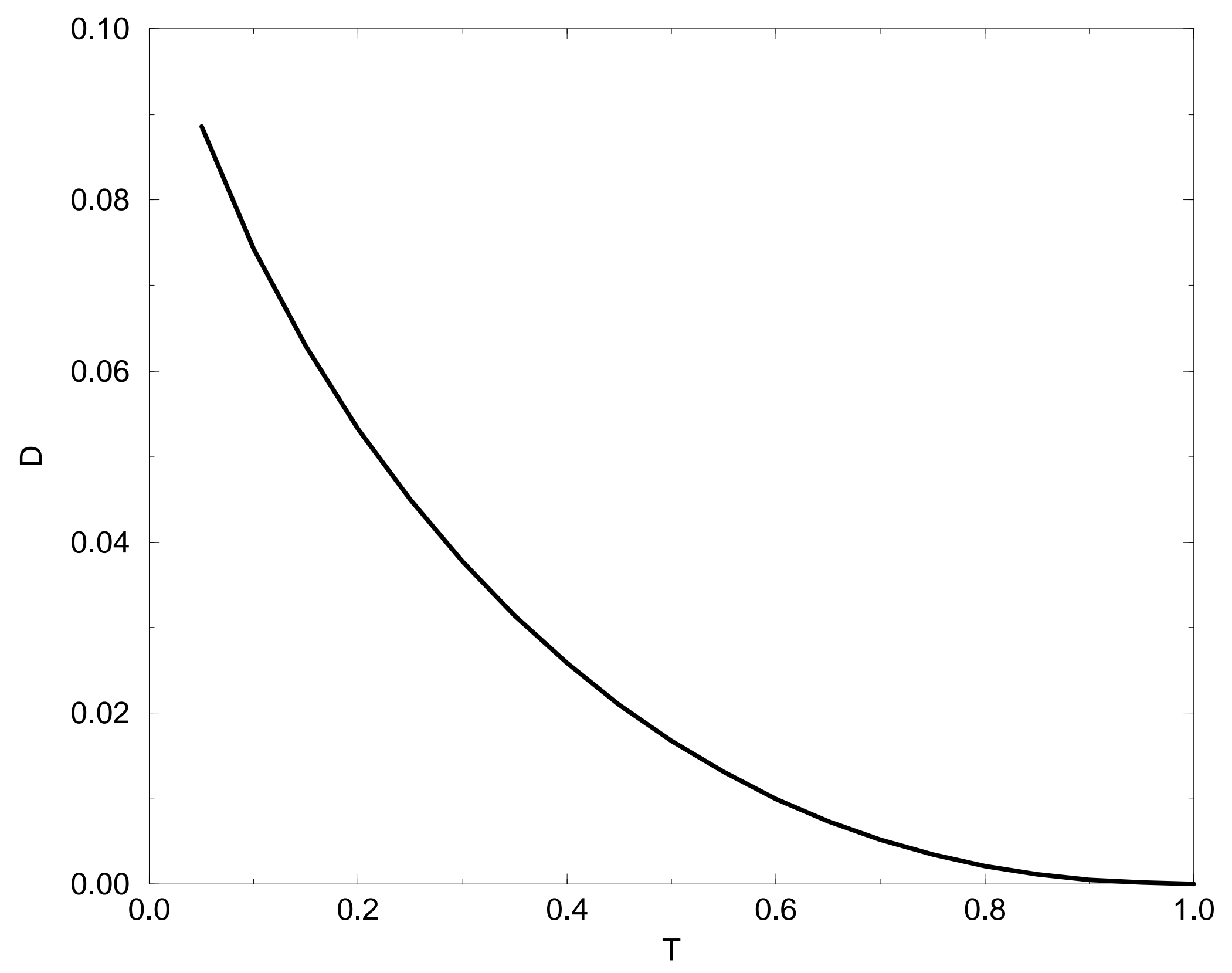


Figure 2

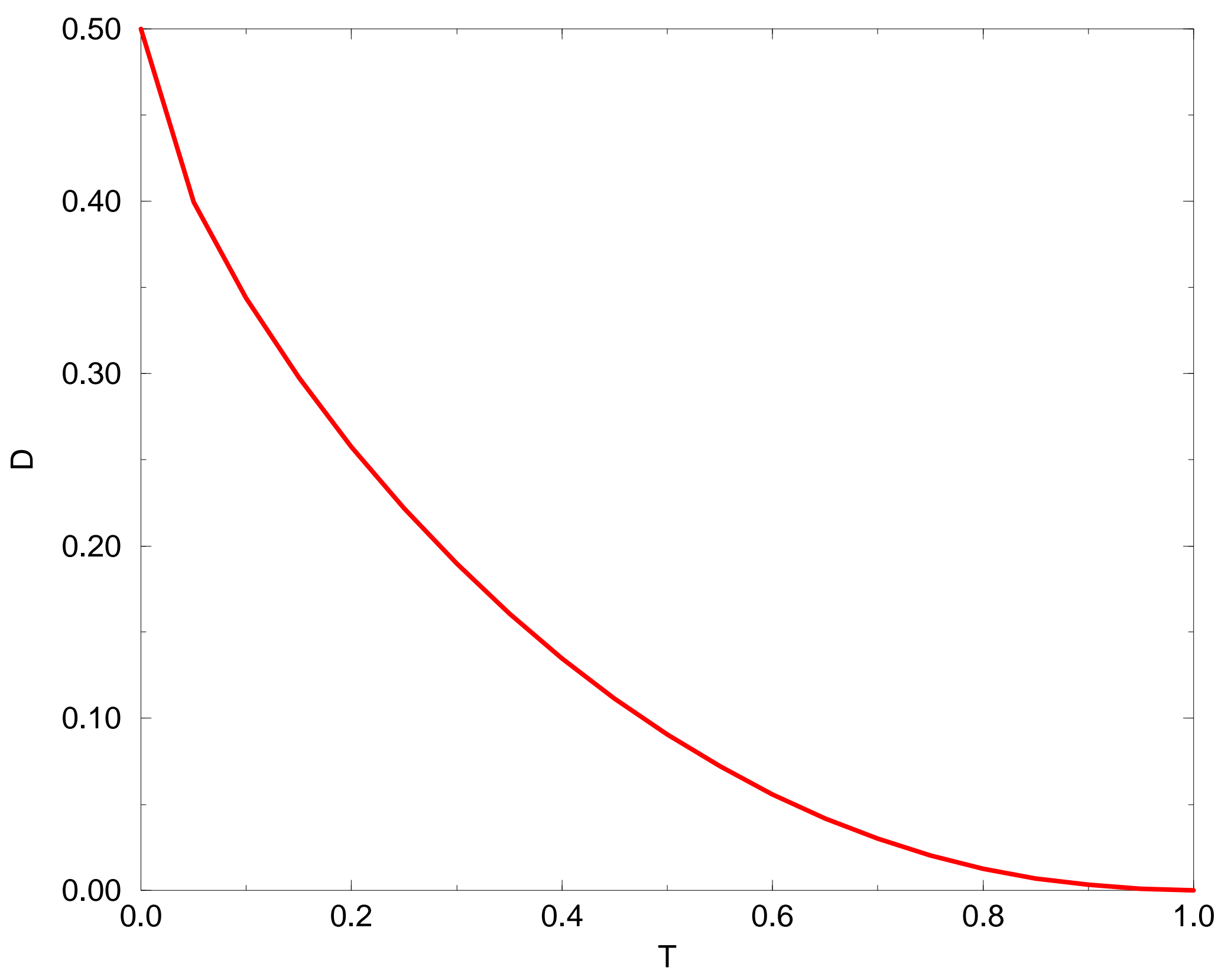

